\title{
On similarity solutions of MHD flow over a nonlinear stretching surface in non-Newtonian power-law fluid
}

\author{
Gabriella Bognár ${ }^{\bowtie}$ \\ University of Miskolc, Miskolc-Egyetemváros, H-3515, Hungary \\ Received 28 July 2015, appeared 11 August 2016 \\ Communicated by Tibor Krisztin
}

\begin{abstract}
Our aim is to discuss the similarity solutions to the MHD flow over a stretching impermeable surface in an electrically conducting fluid in the free stream for nonNewtonian power-law fluid flows. The interest is to examine the existence and nonexistence of solutions and to investigate the influence of the parameters via numerical solutions obtained with Chebyshev spectral method.
\end{abstract}

Keywords: boundary-layer, similarity solutions, existence, non-existence, spectral method.

2010 Mathematics Subject Classification: 34B40, 35G45.

\section{Introduction}

The study of boundary layer flow and its applications are of great importance in many engineering processes, such as in production of wire drawing, paper sheets, plastic foils, crystal growing, cable coating and many others, to get final product of desired quality and characteristics.

Sakiadis [17] was the first who investigated boundary layer flow along a moving continuous plate. Crane gave an exact analytical solution for the steady two-dimensional Newtonian flow problem to a linearly stretching surface whose velocity is linearly proportional to the distance from the slit [10]. A considerable amount of research has been reported on the similarity solutions for moving plates.

The boundary layer flow on a moving permeable plate parallel to a moving stream has been studied by Steinheuer [18], Klemp and Acrivos [16], and later numerically by Ishak et al. [15].

The complex nature of the boundary layer flow under the influence of a magnetic field with the induced magnetic field was considered numerically by Cobble [9] and Soundalgekar et al. [19] for Newtonian media. The MHD flow of a non-Newtonian power-law fluid was studied by constant transverse magnetic field over steady surface by Djukic [11] and Chiam [8].

Our aim is to analyze the similarity solution for boundary layer flow of a non-Newtonian viscous fluid in a potential flow over a stretching elastic flat surface given by $U_{\infty} x^{m}$, where $x$

\footnotetext{
$\bowtie$ Email: v.bognar.gabriella@uni-miskolc.hu
} 
is the coordinate along the plate measured from the leading edge, $m$ and $U_{\infty}$ are constants; moreover, we extend the results given by Chiam [8] on fixed plate to stretching surfaces. Here $\mathrm{x}$ denotes the coordinate along the plate measured from the leading edge $U_{\infty}$ and $m$ are constants.

\section{Mathematical model}

The steady laminar flow of a non-Newtonian electrically conducting incompressible fluid past a two-dimensional body is considered. The velocity components are represented by $u$ and $v$ in the coordinates along and normal to the body surface, $x$ and $y$ directions, respectively. The external velocity distribution is given by $u_{\infty}(x)=U_{\infty} x^{m}$ and the imposed external transverse magnetic field by $B(x)=B_{0} x^{(m-1) / 2}$, where $B_{0}>0, U_{\infty}$ and $m$ are constants [9]. The continuity and momentum equations are given by

$$
\begin{gathered}
\frac{\partial u}{\partial x}+\frac{\partial v}{\partial y}=0 \\
u \frac{\partial u}{\partial x}+v \frac{\partial u}{\partial y}=\frac{K}{\rho} \frac{\partial}{\partial y}\left(\left|\frac{\partial u}{\partial y}\right|^{n-1} \frac{\partial u}{\partial y}\right)+u_{\infty} \frac{\partial u_{\infty}}{\partial y}-\sigma B^{2}\left(u-u_{\infty}\right),
\end{gathered}
$$

where $\rho$ denotes the density, $\sigma$ the electric conductivity and the non-linear model describing the non-Newtonian fluid is

$$
\tau_{x y}=K\left|\frac{\partial u}{\partial y}\right|^{n-1} \frac{\partial u}{\partial y}
$$

with two parameters: the consistency coefficient $K$ and the power-law exponent $n$. The case $0<n<1$ corresponds to pseudoplastic fluids (or shear-thinning fluids), the case $n>1$ is known as dilatant or shear-thickening fluids. For $n=1$, one recovers a Newtonian fluid. The deviation of $n$ from a unity indicates the degree of deviation from Newtonian behavior.

The boundary conditions for impermeable surface with stretching velocity $u_{w}(x)=U_{w} x^{m}$ are the following

(i) at the solid surface $y=0$ neither slip nor mass transfer: $u(x, 0)=u_{w}(x), v(x, 0)=0$,

(ii) outside the viscous boundary layer the streamwise velocity component $u$ should approach $u_{\infty}$ :

$$
\lim _{y \rightarrow \infty} u(x, y)=u_{\infty}(x) .
$$

We apply the concept of similarity solution approach by introducing first the stream function $\psi(x, y)$. Then the velocity components are

$$
u=\frac{\partial \psi}{\partial y}, \quad v=-\frac{\partial \psi}{\partial x}
$$

and the continuity equation (2.1) is automatically satisfied. The momentum equation (2.2) becomes

$$
\frac{\partial \psi}{\partial y} \frac{\partial^{2} \psi}{\partial y \partial x}-\frac{\partial \psi}{\partial x} \frac{\partial^{2} \psi}{\partial y^{2}}=\alpha \frac{\partial}{\partial y}\left(\left|\frac{\partial^{2} \psi}{\partial y^{2}}\right|^{n-1} \frac{\partial^{2} \psi}{\partial y^{2}}\right)+u_{\infty} \frac{\partial u_{\infty}}{\partial x}-\sigma B^{2}\left(\frac{\partial \psi}{\partial y}-u_{\infty}\right),
$$

$\alpha=K / \rho$ and the boundary conditions are

$$
\frac{\partial \psi}{\partial y}(x, 0)=U_{w} x^{m}, \quad \frac{\partial \psi}{\partial x}(x, 0)=0, \quad \lim _{y \rightarrow \infty} \frac{\partial \psi}{\partial y}(x, 0)=U_{\infty} x^{m} .
$$


Applying similarity transformation

$$
\psi(x, y)=b x^{\beta} f(\eta), \quad \eta=d y x^{-\delta}
$$

with parameters $b, d, \beta, \delta$ one reduces (2.3) to the ordinary differential equation

$$
\left(\left|f^{\prime \prime}\right|^{n-1} f^{\prime \prime}\right)^{\prime}+\beta f f^{\prime \prime}+m\left(1-f^{\prime 2}\right)+M\left(1-f^{\prime}\right)=0, \quad \eta \in(0, \infty),
$$

where

$$
\beta=\frac{m(2 n-1)+1}{n+1}, \quad \delta=\frac{m(n-2)+1}{n+1}, \quad b=1, \quad d=U_{\infty}
$$

and $M=\sigma B_{0}^{2} /\left(U_{\infty}\right)$ denotes the magnetic parameter. Here, the prime indicates differentiation with respect to $\eta$. The corresponding boundary conditions (2.4) become

$$
\begin{gathered}
f(0)=0, \quad f^{\prime}(0)=\lambda, \\
\lim _{\eta \rightarrow \infty} f^{\prime}(\eta)=1,
\end{gathered}
$$

with velocity ratio $\lambda=u_{w} / u_{\infty}$. The main interest of the numerical studies is the skin friction when the skin friction parameter $C_{f}$ satisfies

$$
C_{f}=2 R e_{x}^{-1 /(n+1)}\left[\frac{m(2 n-1)+1}{n(n+1)}\right]^{n /(n+1)}|\gamma|^{n-1} \gamma,
$$

where $\gamma=f^{\prime \prime}(0)$ and

$$
\operatorname{Re}_{x}=\frac{u_{w}(x)^{2-n} x^{n}}{\rho}
$$

is the local Reynolds number.

The boundary value problem (2.5), (2.6) and (2.7) is determined by four parameters $n, m$, $M$ and $\lambda$. We notice that for special values of the parameters, equation (2.5) involves several well-known problems investigated by many authors. If $n=1, m=0, M=0, \lambda=0$, the problem is recognized as the famous Blasius problem [3]. The existence of a unique solution has been proved by Weyl [20]. On the base of numerical simulations for $\lambda \neq 0$, Steinheuer [18] and Klemp and Acrivos [16] reported that to the Blasius-equation dual solutions exist as long as $\lambda$ is smaller than the critical value $\lambda_{c}$, after which no similarity solutions exist. For $\lambda<0$, Callegari and Nachman [7] proved the existence of unique solution. For $n=1, m=0, M=0$ and $0<\lambda<\lambda_{c}$ the non-uniqueness of the solution was shown by Hussaini and Lakin [14] and $\lambda_{c}$ was found to be 0.3541 . The numerical calculations indicate that for non-Newtonian fluids $(n \neq 1, m=0, M=0)$, there is also critical value $\lambda_{c}$ such that solution to the boundary layer problem exists only if $\lambda<\lambda_{c}$. Estimation for the critical velocity ratio $\lambda_{c}$ depending on the power-law exponent $n$ was given in [4]. If $n=1, m \neq 0, M=0, \lambda=0$, equation (2.5) corresponds to the Falkner-Skan equation [12]. Numerical solutions for velocity and temperature field in MHD Falkner-Skan flow $(n=1, m \neq 0, M \neq 0, \lambda=0)$ are obtained by Soundalgekar et al. [19]. For $\lambda \neq 0$, Aly et al. investigated the existence of infinite number of solutions in [1]. The non-Newtonian flow $(n \neq 1)$ of power-law fluids in the presence of an arbitrary transverse magnetic field $(m \neq 0, M \neq 0, \lambda=0)$ was studied by Galerkin's method and Crocco variables by Djukic in [11].

The present paper discusses the MHD flow over a stretching impermeable surface in an electrically conducting fluid in the free stream $u_{\infty}(x)$ for non-Newtonian fluid flows $n \neq 1$, $m \neq 0, M \neq 0, \lambda \neq 0$. 


\section{Existence and non-existence of solutions}

The existence of solutions can be established by a shooting method. The boundary condition at infinity (2.7) is replaced by $f^{\prime \prime}(0)=\gamma$, where $\gamma \neq 0$. The task is to determine $\gamma$ such that the corresponding solution satisfies (2.7). Therefore, we consider the initial value problem (IVP)

$$
\begin{gathered}
\left(\left|f^{\prime \prime}\right|^{n-1} f^{\prime \prime}\right)^{\prime}+\frac{m(2 n-1)+1}{n+1} f f^{\prime \prime}+m\left(1-f^{\prime 2}\right)+M\left(1-f^{\prime}\right)=0, \quad \eta \in(0, \infty), \\
f(0)=0, \quad f^{\prime}(0)=\lambda, \quad f^{\prime \prime}(0)=\gamma .
\end{gathered}
$$

Our aim is to derive conditions on the parameters involved in (3.1) such that solution $f_{\gamma}$ is global, i.e., $f_{\gamma}$ exists on the entire positive axis $\mathbf{R}^{+}$and satisfies $f_{\gamma}^{\prime}(\infty)=1$. A local in $\eta$ solution $f_{\gamma}$ exists on $\left(0, \eta_{\gamma}\right), \eta_{\gamma} \leq \infty$, where $\left(0, \eta_{\gamma}\right)$ is the maximal interval of existence. Since $\gamma \in \mathbf{R}$ is arbitrary, problem (3.1) has infinitely many solutions.

Taking the integral of equation in (2.5) with initial conditions, the local solution $f_{\gamma}$ satisfies the following equality

$$
\begin{aligned}
& \left|f_{\gamma}^{\prime \prime}(\eta)\right|^{n-1} f_{\gamma}^{\prime \prime}(\eta)+\beta f_{\gamma}^{\prime}(\eta) f_{\gamma}(\eta)-M f_{\gamma}(\eta) \\
& \quad=\left|f^{\prime \prime}(0)\right|^{n-1} f^{\prime \prime}(0)-(M+m) \eta+\frac{3 n m+1}{n+1} \int_{0}^{\eta} f_{\gamma}^{\prime}(s)^{2} d s
\end{aligned}
$$

for all $0 \leq \eta<\eta_{\gamma}$.

Definition 3.1. Function $f_{\gamma}(\eta)$ is a solution to (3.1) under conditions

(i) $f_{\gamma}(\eta) \in C^{2}(0, \infty)$,

(ii) $\left|f_{\gamma}^{\prime \prime}\right|^{n-1} f_{\gamma}^{\prime \prime} \in C^{1}(0, \infty)$,

(iii) $\lim _{\eta \rightarrow \infty} f_{\gamma}^{\prime}(\eta)=1$ and $\lim _{\eta \rightarrow \infty} f_{\gamma}^{\prime \prime}(\eta)=0$,

moreover, $f_{\gamma}$ satisfies the differential equation and the initial conditions.

Let $f_{\gamma}$ be the local solution of (2.5), we define

$$
E(\eta):=E\left(f_{\gamma}(\eta)\right)=\frac{n}{n+1}\left|f_{\gamma}^{\prime \prime}\right|^{n+1}-\frac{m}{3} f_{\gamma}^{\prime 3}-\frac{M}{2} f_{\gamma}^{\prime 2}+(M+m) f_{\gamma}^{\prime} .
$$

Multiplying equation in (2.5) by $f_{\gamma}^{\prime \prime}$ after integration we obtain

Lemma 3.2. The energy functional $E(\eta)$ defined by (3.3) satisfies

$$
E^{\prime}(\eta)=-\frac{m(2 n-1)+1}{n+1} f_{\gamma} f_{\gamma}^{\prime \prime 2}
$$

on $\left(0, \eta_{\gamma}\right)$.

Note, that using the initial conditions one gets

$$
E(0)=\frac{n}{n+1}|\gamma|^{n+1}+F(\lambda),
$$

with

$$
F(\lambda)=-\frac{m}{3} \lambda^{3}-\frac{M}{2} \lambda^{2}+(M+m) \lambda .
$$


For $m<0$, one gets that $F(\lambda)<0$ for $0<\lambda<\Gamma_{1}$ with

$$
\Gamma_{1}=-\frac{3 M}{4 m}-\sqrt{\left(\frac{3 M}{4 m}\right)^{2}+\frac{3 M}{m}+3}
$$

and $F(\lambda) \geq 0$ for $\lambda \geq \Gamma_{1}$.

The existence of infinitely many solutions to (2.5)-(2.7) was proved for some values of $m$, $n, M$, and $\lambda$ in [2].

Theorem 3.3. For any $M>0, m+M<0, m(2 n-1)+1>0$ and $0<\lambda<\Gamma_{1},\left(\Gamma_{1}>1\right)$ satisfying

$$
|\gamma|^{n+1} \leq(n+1)\left[\frac{1}{3} m \lambda^{3}+\frac{1}{2} M \lambda^{2}-(M+m) \lambda\right]
$$

(i) solution $f_{\gamma}$ is positive and monotonic increasing on $\left(0, \eta_{\gamma}\right)$ and global;

(ii) $\lim _{\eta \rightarrow \infty} f_{\gamma}(\eta)=\infty, \lim _{\eta \rightarrow \infty} f_{\gamma}^{\prime \prime}(\eta)=0$ and $\lim _{\eta \rightarrow \infty} f_{\gamma}^{\prime}(\eta)=1$.

Proof. See [2]. It also gives that $\lim _{\eta \rightarrow \infty} E(\eta)=\frac{M}{2}+\frac{2 m}{3}$, which is negative for $\Gamma_{1}>1$.

Moreover the following non-existence result was established.

Theorem 3.4. Problem (2.5)-(2.7) has no non-negative solution for $M>0, m+M<0$, $m(2 n-1)+1<0$ and $\lambda \geq \Gamma_{1}$.

Proof. See [2].

Theorem 3.5. Problem (2.5)-(2.7) has no non-negative solution for $M>0, m+M<0$, $m(2 n-1)+1<0,0<\lambda<\Gamma_{1}$ and

$$
|\gamma|^{n+1} \geq \frac{n+1}{n}\left(\frac{1}{3} m \lambda^{3}+\frac{1}{2} M \lambda^{2}-(M+m) \lambda\right) .
$$

Proof. Following the paper [1] and [2], let us assume that $f$ is a non-negative solution to (2.5)(2.7). Then $E^{\prime}(\eta)=-\beta f_{\gamma} f_{\gamma}^{\prime \prime 2}$ is non-negative. Therefore, $E$ is monotonic increasing and hence

$$
\begin{gathered}
E(0) \leq \lim _{\eta \rightarrow \infty} E(\eta), \\
\frac{n}{n+1}|\gamma|^{n+1}-\frac{1}{3} m \lambda^{3}-\frac{1}{2} M \lambda^{2}+(M+m) \lambda \leq \frac{M}{2}+\frac{2 m}{3}<0,
\end{gathered}
$$

which contradicts (3.5).

For $m>0$ let us define

$$
\Gamma_{2}=-\frac{3 M}{4 m}+\sqrt{\left(\frac{3 M}{4 m}\right)^{2}+\frac{3 M}{m}+3 .}
$$

We remark that if $\lambda>\Gamma_{2}$ then $F(\lambda)<0$.

Theorem 3.6. For any $M>0, m>0, m(2 n-1)+1>0$ and $\lambda>\Gamma_{2}$ satisfying

$$
|\gamma|^{n+1} \leq \frac{n+1}{n}\left(\frac{1}{3} m \lambda^{3}+\frac{1}{2} M \lambda^{2}-(M+m) \lambda\right)
$$

solution $f_{\gamma}$ to $(2.5)-(2.7)$

(i) is positive and monotonic increasing on $\left(0, \eta_{\gamma}\right)$ and global;

(ii) $\lim _{\eta \rightarrow \infty} f_{\gamma}(\eta)=\infty, \lim _{\eta \rightarrow \infty} f_{\gamma}^{\prime \prime}(\eta)=0$ and $\lim _{\eta \rightarrow \infty} f_{\gamma}^{\prime}(\eta)=1$. 
Proof of (i). Remark that $E(0) \leq 0$ and $E^{\prime}=-\beta f_{\gamma} f_{\gamma}^{\prime \prime 2}$ on $\left(0, \eta_{\gamma}\right)$. As $\lambda>0$ we can assume that $f_{\gamma}$ and $f_{\gamma}^{\prime}$ are positive on some $\left(0, \eta_{0}\right)$, i.e., $E^{\prime} \leq 0$ and $E\left(\eta_{0}\right) \leq E(0)$, which gives

$$
E\left(\eta_{0}\right)<0 .
$$

If $f_{\gamma}^{\prime}\left(\eta_{0}\right)=0$, then $E\left(\eta_{0}\right)=E(0)=0$ for all $0 \leq \eta \leq \eta_{0}$. Then $f_{\gamma}^{\prime \prime} \equiv 0$ on $\left(0, \eta_{0}\right)$ implies $\lambda=0$, and this leads to contradiction. Hence, $f_{\gamma}$ is positive and it is a strictly monotone increasing function.

To show that $f_{\gamma}$ is global we use the energy function $E$, which gives

$$
\frac{n}{n+1}\left|f_{\gamma}^{\prime \prime}\right|^{n+1}-\frac{m}{3} f_{\gamma}^{\prime 3}-\frac{M}{2} f_{\gamma}^{\prime 2}+(M+m) f_{\gamma}^{\prime} \leq \frac{n}{n+1}|\gamma|^{n+1}-\frac{m}{3} \lambda^{3}-\frac{M}{2} \lambda^{2}+(M+m) \lambda .
$$

From this, $f_{\gamma}^{\prime \prime}$ and $f_{\gamma}^{\prime}$ are bounded, therefore $f_{\gamma}$ is also bounded on $\left(0, \eta_{\gamma}\right)$ if $\eta_{\gamma}$ is finite, which is impossible. Then $\eta_{\gamma}$ is infinity and $f_{\gamma}$ is global.

Proof of (ii). First we show that $\lim _{\eta \rightarrow \infty} f_{\gamma}(\eta)=\infty$. Let us assume that $f_{\gamma}$ has a limit at infinity

$$
\lim _{\eta \rightarrow \infty} f_{\gamma}(\eta)=L, \quad L \in(0, \infty]
$$

as $f_{\gamma}^{\prime}$ is positive. Assume that $L$ is finite. Hence, there exists a sequence $\left(\eta_{r}\right)$ converging to infinity with $r$ such that $f^{\prime}\left(\eta_{r}\right)$ tends to 0 as $r \rightarrow \infty$. Then,

$$
-\frac{m}{3} f^{\prime}\left(\eta_{r}\right)^{3}-\frac{M}{2} f^{\prime}\left(\eta_{r}\right)^{2}+(M+m) f^{\prime}\left(\eta_{r}\right) \leq E\left(\eta_{r}\right) \leq E(0)
$$

for any $r \in \mathbf{N}$. It gives $0 \leq E(\infty) \leq E(0)$, a contradiction.

Next, we show that

$$
\lim _{\eta \rightarrow \infty} f_{\gamma}^{\prime \prime}(\eta)=0
$$

which is the case if $f_{\gamma}^{\prime \prime}$ is monotone on some interval $\left[\eta_{0}, \infty\right)$ since $f_{\gamma}^{\prime}$ and $f_{\gamma}^{\prime \prime}$ are bounded. Assume that $\left|f_{\gamma}^{\prime \prime}\right|^{n-1} f_{\gamma}^{\prime \prime}$ is not monotone on any interval $\left[\eta_{0}, \infty\right)$. Then there exists a sequence $\left\{\eta_{r}\right\}$ tending to infinity as $r \rightarrow \infty$ such that $\left(\left|f_{\gamma}^{\prime \prime}\right|^{n-1} f_{\gamma}^{\prime \prime}\right)^{\prime}\left(\eta_{r}\right)=0$, and $\left(\left|f_{\gamma}^{\prime \prime}\right|^{n-1} f_{\gamma}^{\prime \prime}\right)\left(\eta_{2 r}\right)$ is a local maximum, $\left(\left|f_{\gamma}^{\prime \prime}\right|^{n-1} f_{\gamma}^{\prime \prime}\right)\left(\eta_{2 r+1}\right)$ is a local minimum. Applying $\eta=\eta_{r}$ to the differential equation, one gets

$$
\frac{m(2 n-1)+1}{n+1} f_{\gamma}^{\prime \prime}\left(\eta_{r}\right)=-\frac{m\left(1-f_{\gamma}^{\prime 2}\left(\eta_{r}\right)\right)+M\left(1-f_{\gamma}^{\prime}\left(\eta_{r}\right)\right)}{f_{\gamma}\left(\eta_{r}\right)} .
$$

As $f_{\gamma}^{\prime}$ is bounded and $f_{\gamma}$ tends to infinity then $f_{\gamma}^{\prime \prime}\left(\eta_{r}\right) \rightarrow 0$ as $r \rightarrow \infty$ and

$$
\lim _{\eta \rightarrow \infty} f_{\gamma}^{\prime \prime}(\eta)=0
$$

Let $f_{\gamma}$ be the global solution of (3.1), then we show that it satisfies $\lim _{\eta \rightarrow \infty} f_{\gamma}^{\prime}(\eta)=1$. Assume that $f_{\gamma}^{\prime}$ has a finite limit at infinity. Then, function $E$ has a finite limit at infinity, $E(\infty)$. Since $\lim _{\eta \rightarrow \infty} f_{\gamma}^{\prime \prime}(\eta)=0$, then

$$
\lim _{\eta \rightarrow \infty}\left(-\frac{m}{3} f_{\gamma}^{\prime 3}-\frac{M}{2} f_{\gamma}^{\prime 2}+(M+m) f_{\gamma}^{\prime}\right)=E(\infty) .
$$


Let us assume that for two non-negative $L_{1}$ and $L_{2}$

$$
\liminf _{\eta \rightarrow \infty}^{\prime}(\eta)=L_{1}
$$

and

$$
\limsup _{\eta \rightarrow \infty} f_{\gamma}^{\prime}(\eta)=L_{2}
$$

and these satisfy

$$
-\frac{m}{3} L_{i}^{3}-\frac{M}{2} L_{i}^{2}+(M+m) L_{i}=E(\infty), \quad i=1,2 .
$$

We suppose, that $L_{1} \neq L_{2}$ and choose $\bar{L}$ such that $L_{1}<\bar{L}<L_{2}$. Let $\left\{\eta_{r}\right\}_{r \in \mathbf{N}}$ be a sequence tending to infinity with $r$ such that

$$
\lim _{\eta \rightarrow \infty} f_{\gamma}^{\prime}\left(\eta_{r}\right)=\bar{L}
$$

With function $E$, we have

$$
E(\infty)=-\frac{m}{3} \bar{L}^{3}-\frac{M}{2} \bar{L}^{2}+(M+m) \bar{L}
$$

for all $L_{1}<\bar{L}<L_{2}$, which is impossible. Then $L_{1}=L_{2}$. Hence, $f_{\gamma}^{\prime}(\eta)$ has finite limit a infinity. Denote this limit by $\bar{L}$, which is non-negative. Assume that $\bar{L}=0$, then $E(\infty)=0$. Since $E$ is a decreasing function we get $E \equiv 0$ and get a contradiction. Hence $\bar{L}>0$. Next, we use the identity (3.2) to obtain

$$
\begin{aligned}
& \left|f_{\gamma}^{\prime \prime}(\eta)\right|^{n-1} f_{\gamma}^{\prime \prime}(\eta)=-(M+m) \eta-\frac{m(2 n-1)+1}{n+1} \bar{L}^{2} \eta+\frac{1+3 n m}{n+1} \bar{L}^{2} \eta+o(1) \\
& \left|f_{\gamma}^{\prime \prime}(\eta)\right|^{n-1} f_{\gamma}^{\prime \prime}(\eta)=\left[m \bar{L}^{2}+M \bar{L}-(M+m)\right] \eta+o(1)
\end{aligned}
$$

as $\eta \rightarrow \infty$. From this, we deduce that

$$
m \bar{L}^{2}+M \bar{L}-(M+m)=0,
$$

which implies that $\bar{L}=1$ for a positive $\bar{L}$. This ends the proof of Theorem 3.6.

We finish this section with a non-existence result for $m>0$.

Theorem 3.7. Problem (2.5)-(2.7) has no non-negative solution for $m>0, M>0, m(2 n-1)+1<0$, $\lambda>0$ and

$$
|\gamma|^{n+1}>\frac{n+1}{n}(\lambda-1)^{2}\left(\lambda+2+\frac{3 M}{4 m}\right) .
$$

Proof. Let $f_{\gamma}$ be a non-negative solution to (2.5). Function $E$ satisfies

$$
E^{\prime}(\eta)=-\frac{m(2 n-1)+1}{n+1} f f^{\prime \prime 2}
$$

which is non-negative. Clearly,

$$
E(0) \leq \lim _{\eta \rightarrow \infty} E(\eta)
$$

Hence,

$$
\begin{gathered}
\frac{n}{n+1}|\gamma|^{n+1}-\frac{m}{3} \lambda^{3}-\frac{M}{2} \lambda^{2}+(M+m) \lambda \leq \frac{2 m}{3}+\frac{M}{2} \\
|\gamma|^{n+1} \leq \frac{n+1}{n}(\lambda-1)^{2}\left(\lambda+2+\frac{3 M}{4 m}\right)
\end{gathered}
$$

and this contradicts (3.6). 


\section{Numerical solution}

The influence of the parameter values can be investigated through numerical solutions to the above non-Newtonian MHD flow problem. To solve the ordinary differential equation (2.5) under boundary conditions (2.6) and (2.7), we use a Chebyshev spectral method. Spectral methods can be applied to provide very accurate results when the solution is smooth enough. More precisely, if the solution is differentiable to all orders, an exponential (or infinite order or spectral) convergence is achieved. However, if the solution is $m$-times continuously differentiable, the rate of convergence is algebraic: $O\left(k^{m}\right)$, where $k$ is the $k$-th expansion mode [5]. Superior convergence can be achieved for entire functions. In our calculations the collocation method is used. During collocation the function values of the interpolating polynomial at the collocation points (nodal approximation) are determined. For other aspects of the method, we refer to [6]. The $n$-th order Chebyshev polynomial of the first kind, $T_{n}(x)$ is defined on $[-1,1]$. Let us define the modal approximation $I_{N} u(x)$ of a function $u(x)$ as

$$
I_{N} u(x)=\sum_{j=0}^{N} a_{j} T_{j}(x),
$$

where $a_{j}$ are constants, $T_{j}(x)$ are the $j$-th Chebyshev polynomial $(j=0, \ldots, N)$ and the nodal approximation $p_{N} u(x)$ of $u(x)$ can be evaluated in the Lagrange base $l_{j}(x)$ as

$$
p_{N} u(x)=\sum_{j=0}^{N} u_{j} l_{j}(x)
$$

The spectral differentiation for Chebyshev polynomials can be carried out either by a matrix-vector product or by using the Fast Fourier Transform (FFT). We implement the matrixvector multiplication method because of the relatively few number of collocation points. The first derivative of $u$ is approximated as

$$
u^{\prime}(x) \approx \sum_{j=0}^{N} D_{i j} u_{j}, \quad i=0, \ldots, N,
$$

where $\mathbf{D}$ is the first differentiation matrix. Similarly, the $p$-th order derivative is calculated as

$$
\frac{d^{p} u\left(x_{i}\right)}{d x^{p}} \approx \sum_{j=0}^{N} D_{i j}^{(p)} u_{j}, \quad i=0, \ldots, N,
$$

with $\mathbf{D}^{(p)}$ standing for the $p$-th differentiation matrix. For $\mathbf{D}$ and $\mathbf{D}^{(2)}$ exact formulas exist.

One of the methods for solving a boundary value problem on an infinite or semi-infinite interval is the so-called domain truncation. Performing the truncation and the linear mapping we have

$$
\eta \in[0, \infty) \rightarrow \xi[0, L] \stackrel{\varsigma=\frac{\tilde{\xi}}{L}}{\rightarrow} \varsigma \in[0,1] \stackrel{x=2 \varsigma-1}{\rightarrow} x \in[-1,1] .
$$

Introducing $\bar{f}(x)=f(\eta(x))$, boundary value problem (2.5)-(2.7) reads

$$
\begin{gathered}
\left(\frac{2}{L}\right)^{2 n+1}\left(\left|\bar{f}^{\prime \prime}\right|^{n-1} \bar{f}^{\prime \prime}\right)^{\prime}+\left(\frac{2}{L}\right)^{2} \beta \overline{f f}^{\prime \prime}-\left(\frac{2}{L}\right)^{2} m \bar{f}^{\prime 2}-\left(\frac{2}{L}\right) M \bar{f}^{\prime}+m+M=0, \\
\bar{f}(-1)=0, \quad \bar{f}^{\prime}(-1)=\lambda \frac{L}{2}, \quad \bar{f}^{\prime}(1)=\frac{L}{2} .
\end{gathered}
$$




\begin{tabular}{|l|l|c|}
\hline$\lambda$ & $\begin{array}{c}f^{\prime \prime}(0) \\
\text { [spectral] }\end{array}$ & $\begin{array}{c}f^{\prime \prime}(0) \\
{[8]}\end{array}$ \\
\hline 0 & 1.3758 & 1.3759 \\
\hline 0.1 & 1.2036 & \\
\hline 0.2 & 1.0336 & \\
\hline 0.3 & 0.8752 & \\
\hline 0.4 & 0.7171 & \\
\hline 0.5 & 0.5722 & \\
\hline 1 & 0 \\
\hline 1.2 & -0.1792 & \\
\hline 1.5 & -0.6080 & \\
\hline 2 & -1.6520 & \\
\hline
\end{tabular}

(a) $n=0.5$

\begin{tabular}{|l|l|c|}
\hline$\lambda$ & $\begin{array}{c}f^{\prime \prime}(0) \\
\text { [spectral] }\end{array}$ & $\begin{array}{c}f^{\prime \prime}(0) \\
{[8]}\end{array}$ \\
\hline 0 & 1.4992 & 1.4992 \\
\hline 0.1 & 1.3847 & \\
\hline 0.2 & 1.2675 & \\
\hline 0.3 & 1.1458 & \\
\hline 0.4 & 1.0167 & \\
\hline 0.5 & 0.8812 & \\
\hline 1 & 0 & \\
\hline 1.2 & -0.3277 & \\
\hline 1.5 & -0.8506 & \\
\hline 2 & -1.8003 & \\
\hline
\end{tabular}

(b) $n=1.5$

Table 4.1: Variation of $f^{\prime \prime}(0)$ for $m=0, M=2$ and $n=0.5,1.5$.

Let us seek function $g$ such that $\bar{f}(x)=P(x) g(x), P(x)=a x^{2}+b x+c$.

In case of $P(-1)=0, P^{\prime}(-1)=\lambda L / 2$ and $P^{\prime}(1)=L / 2$ are satisfied, $a, b, c$ are obtained as

$$
a=(1-\lambda) L / 8, \quad b=(1+\lambda) L / 4, \quad c=(1+3 \lambda) L / 8 .
$$

Now, the differential equation is reformulated as

$$
\begin{aligned}
& \left(\frac{2}{L}\right)^{2 n+1}\left[6 a g^{\prime}+(6 a x+3 b) g^{\prime \prime}+\left(a x^{2}+b x+c\right) g^{\prime \prime \prime}\right]\left|2 a g+(4 a x+2 b) g^{\prime}+\left(a x^{2}+b x+c\right) g^{\prime \prime}\right|^{n-1} \\
& \quad+\left(\frac{2}{L}\right)^{2} \beta\left[\left(a x^{2}+b x+c\right) g\right]\left[2 a g+(4 a x+2 b) g^{\prime}+\left(a x^{2}+b x+c\right) g^{\prime \prime}\right] \\
& \quad-\left(\frac{2}{L}\right)^{2} m\left[(2 a x+b) g+\left(a x^{2}+b x+c\right) g^{\prime}\right]^{2} \\
& \quad-\left(\frac{2}{L}\right) M\left[(2 a x+b) g+\left(a x^{2}+b x+c\right) g^{\prime}\right]+m+M=0
\end{aligned}
$$

under the boundary conditions

$$
g(-1)=1, \quad g^{\prime}(1)=0
$$

After the discretization of $g(x)$ and its derivatives according to (4.5) and (4.6), the resulting system of nonlinear equations is solved with the Levenberg-Marquardt algorithm in Matlab.

In Table 4.1, we list some values of $f^{\prime \prime}(0)$ for $n=1$ and $m=0.5,1,1.5$ when the magnetic parameter takes 1 and for different values of the velocity ratio in the range $0 \leq \lambda \leq 2$. We note that results published in the literature are special cases of the above. For $\lambda=0$, Chiam [8] obtained numerical solution by shooting method using a fourth-order Runge-Kutta routine. These are in good agreement with our results obtained with spectral method. Two computed skin friction profiles $f^{\prime \prime}(0)\left|f^{\prime \prime}(0)\right|^{n-1}$ are presented in Figure 4.1. The effect of $n$ is opposite if $\lambda<1$ or $\lambda>1$ for shear-thinning $(n=0.5)$ or shear-thickening $(n=1.5)$ fluids.

Figure 4.2 demonstrates that $-\left|f^{\prime \prime}(0)\right|^{n}$ decreases gradually with increasing $n$ in the range [0.5,2] for fixed value of $M$. This observation is consistent with findings of Djukic [11]. 


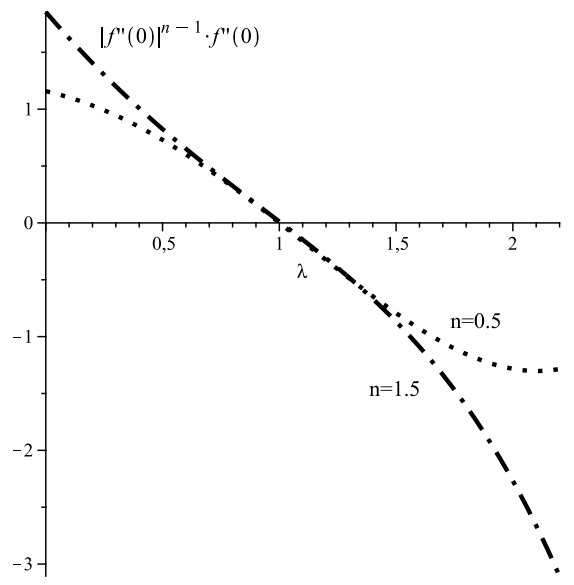

Figure 4.1: Skin friction parameter $f^{\prime \prime}(0)\left|f^{\prime \prime}(0)\right|^{n-1}$ for $n=0.5 ; 1.5$ and $m=0, M=2$.

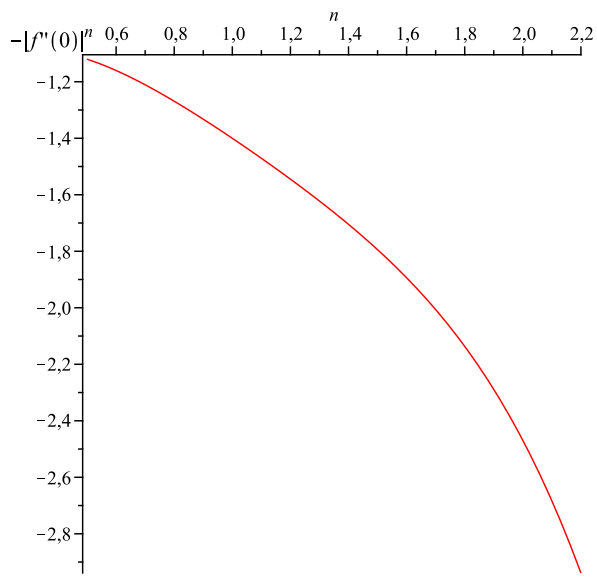

Figure 4.2: Skin friction parameter $-\left|f^{\prime \prime}(0)\right|^{n}$ for $m=0, M=1, \lambda=2$ and $n \in[0.5, \ldots, 2]$.

\begin{tabular}{|l|l|l|}
\hline$\lambda$ & $\begin{array}{c}f^{\prime \prime}(0) \\
\text { [spectral] }\end{array}$ & $\begin{array}{c}f^{\prime \prime}(0) \\
{[19]}\end{array}$ \\
\hline 0 & & \\
\hline 0.2 & 0.8994 & \\
\hline 0.3 & 0.7987 & \\
\hline 0.4 & 0.6947 & \\
\hline 0.5 & 0.5871 & \\
\hline 0.6 & 0.4762 & \\
\hline 0.7 & 0.3620 & \\
\hline 1 & 0 \\
\hline 1.2 & -0.2567 & \\
\hline 1.5 & -0.6642 & \\
\hline 2 & -1.3998 & \\
\hline
\end{tabular}

(a) $m=0$

\begin{tabular}{|l|l|c|}
\hline$\lambda$ & $\begin{array}{c}f^{\prime \prime}(0) \\
\text { [spectral] }\end{array}$ & $\begin{array}{c}f^{\prime \prime}(0) \\
{[19]}\end{array}$ \\
\hline 0 & 1.3599 & 1.3599 \\
\hline 0.2 & 1.1284 & \\
\hline 0.3 & 1.004 & \\
\hline 0.4 & 0.8755 & \\
\hline 0.5 & 0.7414 & \\
\hline 0.6 & 0.6025 & \\
\hline 0.7 & 0.4587 & \\
\hline 1 & 0 & \\
\hline 1.2 & -0.3277 & \\
\hline 1.5 & -0.8506 & \\
\hline 2 & -1.8003 & \\
\hline
\end{tabular}

(b) $m=0.5$

\begin{tabular}{|l|l|c|}
\hline$\lambda$ & $\begin{array}{c}f^{\prime \prime}(0) \\
\text { [spectral] }\end{array}$ & $\begin{array}{c}f^{\prime \prime}(0) \\
{[19]}\end{array}$ \\
\hline 0 & 1.5851 & 1.5851 \\
\hline 0.2 & 1.3190 & \\
\hline 0.3 & 1.1757 & \\
\hline 0.4 & 1.0258 & \\
\hline 0.5 & 0.8696 & \\
\hline 0.6 & 0.7073 & \\
\hline 0.7 & 0.5391 & \\
\hline 1 & 0 & \\
\hline 1.2 & -0.3866 & \\
\hline 1.5 & -1.0052 & \\
\hline 2 & -2.1327 & \\
\hline
\end{tabular}

(c) $m=1$

Table 4.2: Variation of $f^{\prime \prime}(0)$ for $M=1, n=1$ and $m=0,0.5,1$. 


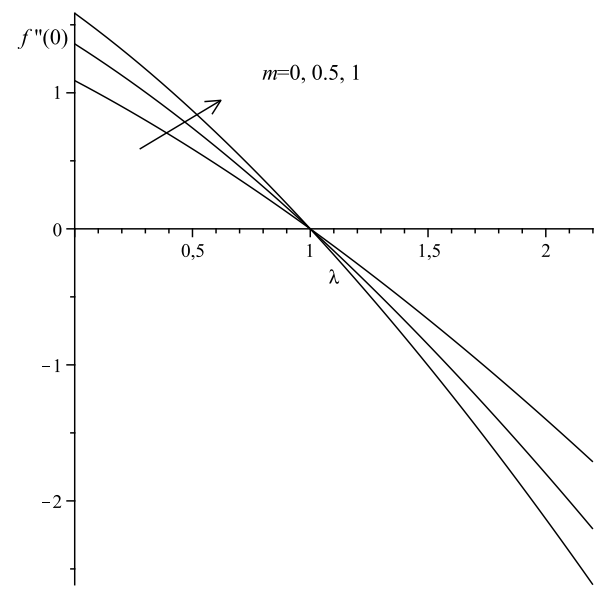

Figure 4.3: Variation of $f^{\prime \prime}(0)$ for $M=1, n=1$ and $m=0,0.5,1$.

The data for $f^{\prime \prime}(0)$ in Table 4.2, which plotted in Figure 4.3 show that $f^{\prime \prime}(0)$ decreases with the velocity ratio $\lambda$ for three different stretching parameter $m$. Our results are in good agreement with those obtained by Soundalgekar et al. [19].

\section{References}

[1] E. A. Aly, M. Benlahsen, M. Guedda, Similarity solutions of a MHD boundarylayer flow past continuous moving surface, Internat. J. Engrg. Sci. 45(2007), 486-503. MR2347973; url

[2] M. Benlahcen, A. Hakim, M. Guedda, Z. Mahani, Similarity solutions of a MHD boundary-layer flow of a non-Newtonian fluid past a continuous moving surface, in: Proc. 12th WSEAS Int. Conf. on Applied Mathematics, Cairo, Egypt, Dec. 29-31, 2007, pp. 7-12. url

[3] H. Blasius, Grenzschichten in Flüssigkeiten mit kleiner Reibung, Z. Math. Phys. 56(1908), $1-37$.

[4] G. BogNÁR, On similarity solutions to boundary layer problems with upstream moving wall in non-Newtonian power-law fluids, IMA J. Applied Math. 77(2012), 546-562. MR10.1093/imamat/hxr033

[5] J. P. Boyd, Chebyshev and Fourier spectral methods, Dover Publications, Inc., Mineola, NY, 2001. MR1874071

[6] C. Canuto, M. Y. Hussaini, A. Quarteroni, T. A. Zang, Spectral methods. Fundamentals in single domains, Springer-Verlag, Berlin, 2006. MR2223552

[7] A. J. Callegari, A. Nachman, Some singular nonlinear differential equations arising in boundary layer theory, J. Math. Anal. Appl. 64(1978), 96-105. MR0478973

[8] T. C. Chiam, Solutions for the flow of a conducting power-law fluid in a transverse magnetic field and with a pressure gradient using Crocco variables, Acta Mech. 137(1999), 225-235. url 
[9] M. Н. СоввLE, Magnetofluiddynamic flow with a pressure gradient and fluid injection, J. Eng. Math. 11(1977), 249-256. url

[10] L. J. Crane, Flow past a stretching plate, Z. Angew. Math. Phys. 2(1970), 645-647. url

[11] Dj. S. DJukic, Hiemenz magnetic flow of power-law fluids, J. Appl. Mech. 41(1974), 822823. url

[12] V. M. FALKNER, S. W. SKan, Some approximate solutions of the boundary layer equations, Phil. Mag. 12(1931), 865-896. ARC-Report 1314.

[13] Z. Hammouch, Multiple solutions of steady MHD flow of dilatant fluids, Eur. J. Pure Appl. Math. 1(2008), 11-20. MR2439804

[14] M. Y. HussainI, W. D. LAKIN, Existence and nonuniqueness of similarity solutions of a boundary-layer problem, Quart. J. Mech. Appl. Math. 39(1986), 177-191. MR827699; url

[15] A. IshaK, R. NAzar, I. Pop, Boundary layer on a moving wall with suction or injection, Chin. Phys. Lett. 8(2007), 2274-2276. url

[16] J. B. Klemp, A. A. Acrivos, A moving-wall boundary layer with reverse flow, J. Fluid Mech. 76(1976), 363-381. url

[17] B. C. SAKIADIS, Boundary layer behavior on continuous solid surfaces. II. The boundary layer on a continuous flat surface, AIChE J. 7(1961), 221-225. url

[18] J. Steinheuer, Die Lösungen der Blasiusschen Grenzschichtdifferentialgleichung, Abh. Braunschweig. Wiss. Gesell. 20(1968), 96-125. MR0241766

[19] V. M. Soundalgekar, H. S. Takhar, M. Singh, Velocity and temperature field in MHD Falkner-Skan flow, J. Phys. Soc. Japan 50(1981), 3139-3143. url

[20] H. WEYL, On the differential equations of the simplest boundary-layer problems, Ann. of Math. (2) 43(1942), 381-407. MR0006294 